
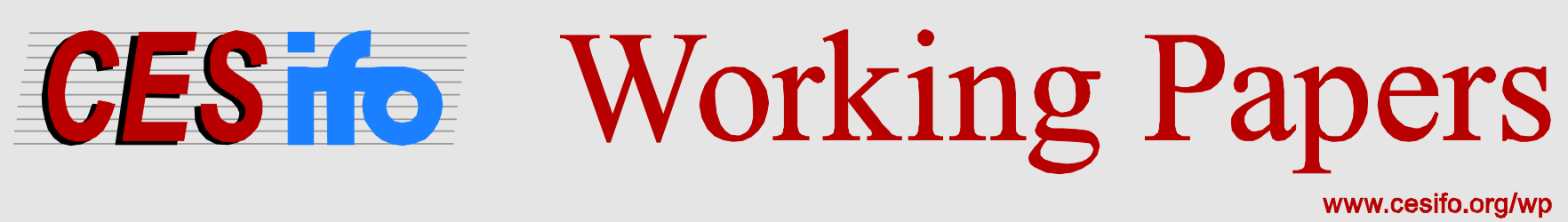

\title{
Cordon Tolling in a City with Congested Bridges
}

\author{
Jan K. Brueckner
}

\author{
CESIFO WORKING PAPER NO. 4898 \\ CATEgory 1: Public FinANCE \\ JULY 2014
}
An electronic version of the paper may be downloaded
- from the SSRN website:
- from the RePEc website:
- from the CESifo website:
wWw.SSRN.com
Www.RePEc.org
www.CESifo-group.org/wp

\section{CESifo}




\title{
Cordon Tolling in a City with Congested Bridges
}

\begin{abstract}
This paper analyzes cordon tolling using a simple model where space is discrete rather than continuous, with commuting costs incurred only on two congested bridges. The first-best regime requires tolls on both bridges, whereas only the inner bridge is tolled under the cordon-toll regime. While less realistic than the continuous-space frameworks used in previous work, this simple setup allows the derivation of a number of analytical results, which were mostly unavailable to authors relying on more-complex models. The paper derives the rule for the optimal cordon toll, showing that it points to a toll level higher than first-best toll on the same bridge. In addition, the analysis shows that cordon tolling leads to a redistribution of population away from the zone immediately outside the cordon, with residents moving to the central zone or to the suburbs. The analysis also shows that the cordon toll raises more revenue than is necessary to pay for the bridge where it is levied, while also tending to increase the capacity of the untolled suburban bridge.
\end{abstract}

JEL-Code: R410.

Keywords: toll, cordon, congestion.

Jan K. Brueckner

Department of Economics

University of California, Irvine

3151 Social Science Plaza

USA - Irvine, CA 92697

jkbrueck@uci.edu

June 2014 


\title{
Cordon Tolling in a City with Congested Bridges
}

by

\author{
Jan K. Brueckner*
}

\section{Introduction}

Road congestion involves a market failure that, when uncorrected, distorts urban landuse. With individual commuters ignoring the congestion externalities they create, perceived commuting costs are too low and commute trips are too long from society's point of view. The result is inefficient urban sprawl, with cities being too spread out.

Congestion pricing serves to internalize the congestion externality, leading to more-compact cities. Analysis of such pricing in the monocentric urban model has a long tradition. Early contributions include Solow (1971) and Mills and de Ferranti (1971), with later work carried out by Kanemoto (1976), Arnott (1979), and Wheaton (1988), among others. In these models, congestion tolls are levied in a continuous fashion across space, so that a commuter pays a separate, spatially dependent toll for each mile traveled on a work trip to the CBD. One result of such a congestion-pricing regime is efficient shrinkage of the city's spatial size.

Real-world implementation of congestion pricing, however, takes a simpler form. The existing toll systems in London, Stockholm, and Singapore all involve some form of "cordon pricing," where a commuter pays a single toll upon entering a zone surrounding the CBD. The border of this zone constitutes a "cordon," the crossing of which triggers payment of a congestion toll. This relatively crude charging system stands in strong contrast to the regime of continuous, spatially differentiated tolls envisioned in the early theoretical work on congestion pricing.

Recognizing this mismatch, a number of recent studies have analyzed the features of an optimal cordon-toll regime, emphasizing that it represents a second-best form of congestion pricing. Mun, Konishi and Yoshikawa (2003) offer the first such analysis in the economics literature, and their work was followed by Verhoef (2005) and more recently by De Lara, de

Palma, Kilani and Piperno (2013). ${ }^{1}$ Mun et al. (2003) use a model without a land market and 
derive analytical conclusions, while the latter two papers rely on numerical analysis applied to the standard urban model. The common goal of all three papers is to find the best location for the cordon and the optimal size of the cordon toll. The latter two papers show that, like a first-best system of continuous congestion tolls, cordon tolling shrinks the spatial size of the city. In addition, all the papers show that the welfare gain from an optimally chosen cordon toll can be surprisingly close to that generated by the first-best regime.

One limitation of the studies by Verhoef (2005) and De Lara et al. (2013), which arises from the complexity inherent in a continuous representation of space in the presence of a land market, is the need to rely heavily on numerical analysis to generate results. As a consequence, these studies offer few purely analytical insights into the cordon-tolling problem in a land-market context. Moreover, the analytical results of Mun et al. (2003), while useful, are derived in the absence of land market. In view of these limitations, the purpose of the present paper is to analyze cordon tolling in the presence of a land market using a simple setting in which a number of analytical results can be derived. The setting is the one by used Brueckner (2014), which was in turn adapted from Brueckner and Helsley (2011), and it substitutes a discrete spatial setup for the continuous space used in previous papers. In particular, the city occupies three islands connected by two congestible bridges, as seen in Figure 1. The CBD is located on the leftmost island, which also contains residences, while the "midcity" and suburban islands are purely residential. The edge of the city lies in the suburban zone, with vacant land outside it. The suburban bridge connects the suburbs to the midcity zone, while the midcity bridge connects that zone to the center. Suburban residents must cross both bridges to reach jobs in the CBD, while midcity residents need only cross the midcity bridge (central residents are not bridge users). Intrazone travel costs are zero, so that the only commuting costs are incurred on the congested bridges. Travel demand is completely inelastic, so that bridge traffic levels are influenced only by the endogenous distribution of the population across the city's zones.

Under the first-best regime, congestion tolls are levied on both the suburban and midcity bridges. With space measured in discrete fashion, cordon tolling has a very a simple representation in this framework. Under a cordon-toll regime, only a single toll is charged, being levied on the midcity bridge, which is closest to the CBD. The toll on the suburban bridge is 
constrained to be zero. ${ }^{2}$

The purpose of the analysis in the paper is to characterize the resulting second-best optimum, comparing it to the outcome under the first-best regime. Unlike in the previous papers, the exact location of the cordon is not an issue in the analysis. But a major contribution of the paper is derivation of the rule that characterizes the level of the optimal cordon toll. The rule shows that the cordon toll is given by the first-best toll expression for the midcity bridge plus a positive term whose magnitude is endogenous. This difference in toll rules suggests that cordon toll that is actually charged on the midcity bridge will be larger than the first-best toll on levied on that bridge. But, since the toll rules contain endogenous quantities that differ between the regimes, this conclusion cannot be established in general but must be affirmed by investigating numerical examples, which show the expected result.

The analysis also investigates the differences in the population distributions across zones under cordon tolling and the first-best regime, with the goal of comparing the city's spatial sizes in the two cases. The one available analytical result establishes that cordon tolling leads to a redistribution of population away from the zone immediately outside the cordon, with residents moving to the central zone or the suburban zone. However, since the comparison of the city's spatial sizes requires comparing the suburban populations under the two regimes, this conclusion leaves the comparison ambiguous. But numerical results show that, while making the city more compact than in the untolled equilibrium, cordon tolling leaves it more spread out than under the first-best regime, a conclusion also reached by Verhoef (2005) and De Lara et al. (2013). Finally, the last part of the analysis introduces bridge capacity choices under the conditions of the self-financing theorem. The analysis demonstrates that revenue from the cordon toll more than pays for the capacity of the midcity bridge, while showing that the capacity rule for the untolled suburban bridge points to a larger capacity than under the first-best regime.

The plan of the paper is as follows. The next section analyzes the first-best toll regime. Section 3 analyzes the cordon-toll regime, while section 4 adds bridge capacity choices to both regimes. Second 5 offers conclusions. 


\section{First-Best Regime}

The variables in the model are as follows. Individual land consumption is denoted $q$, with consumption in the suburban, midcity, and central zones denoted by $q_{s}, q_{m}$, and $q_{c}$. Land rent is equal to the land's fixed opportunity $\operatorname{cost} \bar{r}$ (the agricultural rent) throughout the suburban zone, while land rents in the midcity and suburban zones are given by $r_{m}$ and $r_{c}$, respectively. The nonland composite good, which is numeraire, is denoted $e$, and consumption levels in the three zones are given by $e_{i}, i=s, m, c$. To simplify the analysis, preferences are assumed to take the quasi-linear form $e+v(q)$, where $v^{\prime}>0, v^{\prime \prime}<0$. The populations of the three zones are $n_{i}, i=s, m, c$, and they must sum to the city's fixed overall population $N$, so that

$$
n_{s}+n_{m}+n_{c}=N \text {. }
$$

The cost of crossing a bridge depends on the level of congestion, which is determined by the traffic volume and the bridge's capacity. Bridge capacities are fixed until later in the analysis and are thus suppressed, with an individual commuter's costs of crossing the suburban and midcity bridges given by $t\left(n_{s}\right)$ and $t\left(n_{s}+n_{m}\right)$, respectively. To avoid inessential complications, the fixed bridge capacities are assumed to be equal, so that the same cost function $t(\cdot)$ applies to both bridges. This function satisfies $t^{\prime}>0$ and $t^{\prime \prime} \geq 0$.

In Brueckner and Helsley (2011) and Brueckner (2014), the planner's goal was to minimize the city's resource consumption while generating a fixed common utility level $u$ for the city's residents. However, analysis of the second-best problem involved in cordon tolling requires a different approach. Following Pines and Sadka (1985), the planner is portrayed as maximizing the city's utility level $u$ subject to a resource constraint and other feasibility constraints. While the two approaches are clearly equivalent in characterizing the first-best outcome, the latter approach facilitates the second-best analysis. In addition, the planner is viewed as choosing land rents optimally, rather than choosing consumption levels, an approach that is more illuminating in the second-best problem.

In choosing their land consumption levels, central and midcity consumers satisfy $v^{\prime}\left(q_{i}\right)=r_{i}$ for $i=c, m$, yielding consumption levels of $q_{c}=q\left(r_{c}\right)$ and $q_{m}=q\left(r_{m}\right)$, where $q(\cdot)=v^{\prime-1}(\cdot)$, 
with $q^{\prime}<0$. In contrast to the endogeneity of $q_{c}$ and $q_{c}$, suburban land consumption is fixed at $q_{s}=q(\bar{r})$, a consequence of the exogeneity of $\bar{r}$.

Since it is inefficient to leave vacant land inside the city, the central and midcity zones will be fully occupied, with vacant land found only in the suburban zone. Normalizing the individual land areas of the two inner zones to 1, the constraints

$$
n_{c} q\left(r_{c}\right)=1, \quad n_{m} q\left(r_{m}\right)=1
$$

must be satisfied. Since the total occupied land area in the suburban zone is $n_{s} q(\bar{r})$, the spatial size of the city is then $2+n_{s} q(\bar{r})$. With $q(\bar{r})$ fixed, the city's spatial size thus depends solely on $n_{s}$, the population of the suburban zone.

Imposing the two constraints in (2) and the overall population constraint, the Lagrangean expression for the planner's utility maximization problem is

$$
\begin{aligned}
& u+ \mu_{c}\left(1-n_{c} q\left(r_{c}\right)\right)+\mu_{m}\left(1-n_{m} q\left(r_{m}\right)\right)+\theta\left[N-\left(n_{s}+n_{m}+n_{c}\right)\right] \\
&+\lambda\left[I-\left\{n_{c}\left[u-v\left(q\left(r_{c}\right)\right)\right]+n_{m}\left[u-v\left(q\left(r_{m}\right)\right)\right]+n_{s}[u-v(q(\bar{r}))]\right.\right. \\
&\left.\left.\left.\quad+\left[n_{c} q\left(r_{c}\right)\right)+n_{m} q\left(r_{m}\right)+n_{s} q(\bar{r})\right] \bar{r}+n_{s} t\left(n_{s}\right)+\left(n_{m}+n_{s}\right) t\left(n_{m}+n_{s}\right)\right\}\right],
\end{aligned}
$$

where $\mu_{m}, \mu_{c}, \theta$, and $\lambda$ are Lagrange multipliers and where the expression in brackets in the last two lines (when set equal to zero) represents the city's resource constraint. To understand this constraint, note first that the expression $u-v\left(q\left(r_{c}\right)\right)$ equals $e_{c}$, nonland consumption in the central zone, with a parallel statement applying to the other similar expressions. Therefore, the three terms in the second line equal total nonland consumption, $n_{c} e_{c}+n_{m} e_{m}+n_{s} e_{s}$. The bracketed term in the last line of (3), when multiplied by $\bar{r}$, gives the opportunity cost of the land occupied by the city. The final two terms in (3) give the total commuting cost incurred on the two bridges, while $I$ gives the exogenous endowment of the nonland good available to the city. This endowment can be viewed as coming from a fixed wage income $y$ earned by each resident in CBD employment, so that $I=N y$. 
It is important to note that, since the resource constraint indicates that the outlay for land involves only the opportunity cost, the constraint implicitly assumes that the city residents, acting through a city corporation, acquire land from absentee owners at $\bar{r}$ per unit and rent the land to themselves. The residents earn equal per capita shares of total rental income, so that rental outlays and rental income in excess of $\bar{r}$ cancel in the aggregate, therefore not appearing in the resource constraint. In the language of Pines and Sadka (1986), the city is fully closed. Similarly, the congestion tolls that are needed to support the optimum appear nowhere in the resource constraint. The reason is that the tolls are levied and the revenue then returned to the city's residents in lump-sum fashion by the local government, so that they do not appear in the constraint.

The first-order condition for $u$ is $1-\lambda\left(n_{c}+n_{m}+n_{s}\right)=0$, or $\lambda=1 / N>0$, given (1). Note that $\lambda$, being the multiplier on the resource constraint, gives the marginal utility from an extra unit of endowment. The first-order conditions for $r_{c}$ and $r_{m}$ are

$$
\lambda n_{i} q^{\prime}\left(r_{i}\right)\left[v^{\prime}\left(q\left(r_{i}\right)\right)-\bar{r}\right]-\mu_{i} n_{i} q^{\prime}\left(r_{i}\right)=0, \quad i=c, m
$$

Canceling $n_{i} q^{\prime}\left(r_{i}\right)$ and recognizing that $v^{\prime}\left(q\left(r_{i}\right)\right) \equiv r_{i}, i=c, m,(4)$ reduces to

$$
r_{i}=\bar{r}+\frac{\mu_{i}}{\lambda}, \quad i=c, m
$$

This condition says that a zone's land rent equals the opportunity cost $\bar{r}$ plus a premium equal to the shadow price of land in the zone in terms of the nonland good (the shadow price $\mu_{i}$ divided by the marginal utility of extra endowment).

The first-order condition for $n_{c}$ is

$$
-\theta / \lambda=u-v\left(q\left(r_{c}\right)\right)+\bar{r} q\left(r_{c}\right)+\frac{\mu_{c} q\left(r_{c}\right)}{\lambda}=u-v\left(q\left(r_{c}\right)\right)+r_{c} q\left(r_{c}\right),
$$

where (5) has been used. Note that the rightmost expression in (6) equals individual consumption expenditure in the central zone (recall $e_{c}=u-v\left(q\left(r_{c}\right)\right)$ ). With expenditure positive, $\theta<0$ 
must then hold, a natural conclusion since $\theta$ gives the utility change from an increase in $N$, which must be negative.

While a change in $n_{c}$ generates no changes in commuting cost, as seen in (6), differentiating the Lagrangean with respect to $n_{m}$ yields a commuting-cost effect. The first-order condition for $n_{m}$ is then

$$
\begin{aligned}
-\theta / \lambda & =u-v\left(q\left(r_{m}\right)\right)+\bar{r} q\left(r_{m}\right)+\frac{\mu_{m} q\left(r_{m}\right)}{\lambda}+t\left(n_{m}+n_{s}\right)+\left(n_{m}+n_{s}\right) t^{\prime}\left(n_{m}+n_{s}\right) \\
& =u-v\left(q\left(r_{m}\right)\right)+r_{m} q\left(r_{m}\right)+t\left(n_{m}+n_{s}\right)+\left(n_{m}+n_{s}\right) t^{\prime}\left(n_{m}+n_{s}\right)
\end{aligned}
$$

where (5) is again used.

With the RHS of (6) equal to consumption expenditure in the central zone, it follows that $-\theta / \lambda$ equals the common income level of city residents, which includes wage and rental income and any government transfers. But with the LHS of (7) then equal to income, the equation implies that, beyond outlays on $e$, land, and commuting cost $t\left(n_{m}+n_{s}\right)$ (the first three RHS terms), an additional component of expenditure is the expression $\left(n_{m}+n_{s}\right) t^{\prime}\left(n_{m}+n_{s}\right)$. This expression represents the congestion toll that must be levied on midcity residents to generate the first-best optimum. As usual, the toll equals the increase in individual cost generated by an extra commuter $\left(t^{\prime}\right)$ times the number of commuters affected $\left(n_{m}+n_{s}\right)$.

Similarly, the first-order condition for $n_{s}$ reduces to

$$
-\theta / \lambda=u-v(q(\bar{r}))+\bar{r} q(\bar{r})+t\left(n_{m}+n_{s}\right)+\left(n_{m}+n_{s}\right) t^{\prime}\left(n_{m}+n_{s}\right)+t\left(n_{s}\right)+n_{s} t^{\prime}\left(n_{s}\right) .
$$

This condition says that, in addition to incurring $t\left(n_{m}+n_{s}\right)+t\left(n_{s}\right)$ in commuting costs on the midcity and suburban bridges, suburban residents make an additional outlay of $\left(n_{m}+\right.$ $\left.n_{s}\right) t^{\prime}\left(n_{m}+n_{s}\right)+n_{s} t^{\prime}\left(n_{s}\right)$, which represents the congestion tolls levied on the two bridges.

The planning problem has ten unknowns: $u, r_{c}, r_{m}, n_{c}, n_{m}, n_{s}$, and the four multipliers. Solutions are determined by the nine equations consisting of (1), the two equations in (2), the two equations in (5), (6), (7), (8), the resource constraint in (3), and $\lambda=1 / N$. 


\section{Second-Best Cordon-Toll Regime}

\subsection{Setup and first-order conditions}

Under cordon tolling, only a single toll is levied in the city. The toll on the suburban bridge is constrained to be zero, with the toll on the midcity bridge constituting the cordon toll. This zero-toll constraint can be introduced in the model via a requirement that forces toll outlays on the suburban bridge to be zero. Letting $T$ denote the cordon toll, this requirement is embodied in the following constraint:

$u-v\left(q\left(r_{m}\right)\right)+r_{m} q\left(r_{m}\right)+t\left(n_{m}+n_{s}\right)+T=u-v(q(\bar{r}))+\bar{r} q(\bar{r})+t\left(n_{m}+n_{s}\right)+T+t\left(n_{s}\right)$.

The LHS of (9) is total expenditure for a midcity resident, while the RHS is total expenditure for a suburban resident, an expression that does not include a toll on the suburban bridge (only the private cost of crossing that bridge, $t\left(n_{s}\right)$, is present). With equal incomes, these expressions must be the same. Canceling $u, t\left(n_{m}+n_{s}\right)$, and $T$ and appending a Lagrange multiplier $\tau$, the following term is then added to the Lagrangean expression (3) for the firstbest problem:

$$
\tau\left\{r_{m} q\left(r_{m}\right)-v\left(q\left(r_{m}\right)\right)-\left[\bar{r} q(\bar{r})-v(q(\bar{r}))+t\left(n_{s}\right)\right]\right\}
$$

With only $r_{m}$ and $n_{s}$ present in (11), the first-order conditions for the remaining choice variables are unaffected. However, the condition for $r_{m}$ becomes

$$
\lambda n_{m} q^{\prime}\left(r_{m}\right)\left[-v^{\prime}\left(q\left(r_{m}\right)\right)+\bar{r}\right]+\mu_{m} n_{m} q^{\prime}\left(r_{m}\right)+\tau\left\{\left[-v^{\prime}\left(q\left(r_{m}\right)\right)+r_{m}\right] q^{\prime}\left(r_{m}\right)+q\left(r_{m}\right)\right\}=0
$$

Recognizing again that $v^{\prime}\left(q\left(r_{m}\right)\right) \equiv r_{m}$ and rearranging (12), the condition reduces to

$$
r_{m}=\bar{r}+\frac{\mu_{m}}{\lambda}-\frac{\tau q\left(r_{m}\right)}{\lambda n_{m} q^{\prime}\left(r_{m}\right)} .
$$

Since $\tau$ is negative, as shown in the appendix, while $q^{\prime}<0$ holds, last term in (13) is positive. Therefore, the rule giving the second-best midcity land-rent solution points to a smaller value 
than the analogous first-best rule (compare (5)). However, although $\lambda=1 / N$ holds in both the first-best and cordon-toll regimes, the magnitude of the $\mu_{m}$ multiplier will differ between the regimes. As a result, (13) does not allow the levels of $r_{m}$ that actually emerge to be compared. Subsequent analysis shows, however, that the value of $r_{m}$ is indeed lower under cordon tolling.

The first-order condition for $n_{s}$ is given by (8) with the LHS replaced by $-\left[\theta+\tau t^{\prime}\left(n_{s}\right)\right] / \lambda$. The second-best problem has one additional unknown, the multiplier $\tau$, but the new constraint together with the previous conditions (modified in the case of (8) and (13)) yield the secondbest solutions.

\subsection{The cordon toll}

The magnitude of the cordon toll $T$ is of central interest, and optimality conditions can be manipulated to provide an expression for $T$. To do so, note from (13) that the term $\bar{r}+\mu_{m} / \lambda$ is no longer equal to $r_{m}$ but instead equals

$$
r_{m}+\frac{\tau q\left(r_{m}\right)}{\lambda n_{m} q^{\prime}\left(r_{m}\right)}
$$

Therefore, substituting in the first line of (7), expenditure by a midcity resident is

$$
u-v\left(q\left(r_{m}\right)\right)+r_{m} q\left(r_{m}\right)+t\left(n_{m}+n_{s}\right)+\left(n_{m}+n_{s}\right) t^{\prime}\left(n_{m}+n_{s}\right)+\frac{\tau q\left(r_{m}\right)^{2}}{\lambda n_{m} q^{\prime}\left(r_{m}\right)} .
$$

The toll outlay of this resident is captured by the last two terms in (15). As a result, the optimal cordon toll is given by

$$
T=\left(n_{m}+n_{s}\right) t^{\prime}\left(n_{m}+n_{s}\right)+\frac{\tau q\left(r_{m}\right)^{2}}{\lambda n_{m} q^{\prime}\left(r_{m}\right)}
$$

Since $\tau, q^{\prime}<0$, the second term in (16) is positive. Its magnitude depends in part on the size of the multiplier $\tau$, which measures the negative welfare effect of restricting the suburban-bridge toll to be zero (a larger impact calls for a larger cordon toll). Since the first term in (16) is the first-best toll, the following important conclusion can be stated: 
Proposition 1. The cordon-toll rule points to a higher toll than the first-best toll rule for the midcity bridge. The difference in the actual toll levels, however, depends on other endogenous values under the two regimes.

To understand the second part of the proposition, suppose that $n_{m}+n_{s}$ were larger under cordon tolling than in the first-best regime. Then, since the second term in (16) is positive and $n t^{\prime}(n)$ is increasing in $n$, the optimal cordon toll would be higher than the first-best toll on the midcity bridge. But if $n_{m}+n_{s}$ were instead smaller at the second-best optimum, the relationship between the toll levels would be ambiguous. Nevertheless, an outcome where the cordon toll ends up being lower than the first-best midcity toll would appear to be implausible, given the difference in the toll rules. Thus, as intuition would suggest, the inability to levy a toll on the suburban bridge is likely to raise the toll charged on the midcity bridge. The numerical example presented below confirms this view.

The analysis of Mun et al. (2003) generates results similar to Proposition 1. They show that, at locations just inside an optimally located cordon, commuting is underpriced (with congestion costs uninternalized), while commuting is overpriced just outside the cordon, with the optimal cordon toll charging more than external congestion costs at these locations. This latter result is similar to the conclusion stated in the proposition, although it comes from a very different model.

\subsection{First-best and second-best population distributions}

As Proposition 1 makes clear, the population distributions will differ between the two tolling regimes, and one important question is whether the city is more or less spread out under cordon tolling than under the first-best regime. As explained in the introduction, firstbest tolls serve to efficiently restrain the spatial size of the city, reducing $n_{s}$ relative to the equilibrium outcome without tolls. This conclusion is established for the present model in the second part of the appendix. Unfortunately, whether cordon tolling reduces or increases the city's spatial size relative to the first-best regime is ambiguous in general. However, the

following analysis establishes a more-limited result, showing that the population $n_{m}$ of the midcity zone is smaller under cordon tolling. Since the difference in $n_{c}$ is ambiguous, however, no conclusion about the magnitudes of $n_{s}=N-n_{c}-n_{m}$ under the two regimes can be stated. 
The analysis is carried out by focusing on the land consumption levels $q_{c}$ and $q_{m}$ rather than the rent levels $r_{c}$ and $r_{m}$. Using this approach, the condition that equates expenditures in the suburban and midcity zones can be written

$$
\begin{gathered}
u-v\left(q_{s}\right)+v^{\prime}\left(q_{s}\right) q_{s}+t\left(n_{m}+n_{s}\right)+\left(n_{m}+n_{s}\right) t^{\prime}\left(n_{m}+n_{s}\right)+t\left(n_{s}\right)+n_{s} t^{\prime}\left(n_{s}\right)+\delta_{m}+\delta_{s}= \\
u-v\left(q_{m}\right)+v^{\prime}\left(q_{m}\right) q_{m}+t\left(n_{m}+n_{s}\right)+\left(n_{m}+n_{s}\right) t^{\prime}\left(n_{m}+n_{s}\right)+\delta_{m} .
\end{gathered}
$$

Note first that expenditure on land is written as $v^{\prime}\left(q_{i}\right) q_{i}$, recognizing that $v^{\prime}\left(q_{i}\right)$ equals land rent in zone $i$. In addition, note that the terms $\delta_{m}$ and $\delta_{s}$ capture whether cordon tolling or the first-best regime is in place. Under the latter regime, $\delta_{m}=\delta_{s}=0$ holds, while $\delta_{m}>0$ holds under cordon tolling, given (16). In addition, $\delta_{s}<0$ must also hold so as to eliminate the suburban toll expression $n_{s} t^{\prime}\left(n_{s}\right)$ in (17). Thus, a shift to cordon tolling corresponds to an increase in $\delta_{m}$ and a decrease in $\delta_{s}$, starting at values of zero. The analogous condition that equates expenditures in the central and midcity zones is

$$
u-v\left(q_{c}\right)+v^{\prime}\left(q_{c}\right) q_{c}=u-v\left(q_{m}\right)+v^{\prime}\left(q_{m}\right) q_{m}+t\left(n_{m}+n_{s}\right)+\left(n_{m}+n_{s}\right) t^{\prime}\left(n_{m}+n_{s}\right)+\delta_{m} .
$$

Cancelling terms and using (1) and (2) to substitute $n_{s}=N-1 / q_{c}-1 / q_{m}$ and $n_{m}+n_{s}=$ $N-1 / q_{c},(17)$ and (18) can be rewritten as

$$
\begin{gathered}
-v\left(q_{s}\right)+v^{\prime}\left(q_{s}\right) q_{s}+t\left(N-1 / q_{c}-1 / q_{m}\right)+\left(N-1 / q_{c}-1 / q_{m}\right) t^{\prime}\left(N-1 / q_{c}-1 / q_{m}\right)+\delta_{s} \\
-\left[-v\left(q_{m}\right)+v^{\prime}\left(q_{m}\right) q_{m}\right]=0 \\
-v\left(q_{c}\right)+v^{\prime}\left(q_{c}\right) q_{c}-\left[-v\left(q_{m}\right)+v^{\prime}\left(q_{m}\right) q_{m}+t\left(N-1 / q_{c}\right)+\left(N-1 / q_{c}\right) t^{\prime}\left(N-1 / q_{c}\right)+\delta_{m}\right]=0 .
\end{gathered}
$$

With $q_{s}$ fixed from above, (19) and (20) determine solutions for $q_{c}$ and $q_{m}$ conditional on $\delta_{m}$ and $\delta_{s}$.

It can be shown that, when graphed in $\left(q_{m}, q_{c}\right)$ space, $(20)$ yields an upward-sloping curve, with $\partial q_{c} / \partial q_{m}^{(20)}>0$, and (19) yields a downward-sloping curve, with $\partial q_{c} / \partial q_{m}^{(19)}<0$. In addition, an increase in $\delta_{m}$ (movement to the cordon reqime) shifts the curve from (20) downward, 
so that $\partial q_{c} / \partial \delta_{m}^{(20)}<0$ holds, and a decrease in $\delta_{s}$ (movement to the cordon regime) shifts the curve from (19) upward, so that $\partial q_{c} / \partial \delta_{s}^{(19)}>0$ holds. These conclusions make use of the facts that $n+n t^{\prime}(n)$ is increasing in $n$ and $-v(q)+v^{\prime}(q) q$ is decreasing in $q$.

Figure 2 shows this information, with the intersections between the curves in the firstbest and cordon-toll regimes, respectively, illustrating $q_{c}$ and $q_{m}$ differences between regimes (the curves are drawn as linear for simplicity). The no-toll outcomes shown in the figure are discussed below. Note that, since $\delta_{m}$ and $\delta_{s}$ are in fact endogenous quantities when they are non-zero, the actual solutions in the cordon-toll case are not pinned down in the diagram (doing so would require knowledge of the $\delta$ values at the solution). But the fact that, whatever their actual values, $\delta_{m}$ and $\delta_{s}$ are respectively positive and negative under cordon tolling allows a comparison of outcomes under the two regimes. As can be seen from the figure, the solution under cordon tolling must lie to the right of the first-best solution. As result, $q_{m}$ is larger, and thus $n_{m}=1 / q_{m}$ is smaller under cordon tolling than under the first-best regime. However, as can be seen from Figure 1, $q_{c}$ could be larger or smaller under the cordon-toll regime, depending on the exact positions of the curves. Given this ambiguity, the value of $n_{s}=N-1 / q_{c}-1 / q_{m}$ cannot be compared between the regimes, precluding a comparison of the city's spatial sizes in the two cases. A formal comparative-static analysis of (19) and (20), which is equivalent to the demonstration in Figure 2, affirms these conclusions.

Summarizing yields

Proposition 2. The population of the midcity zone is smaller under cordon tolling than under the first-best regime. However, the central populations under the two regimes, as well the populations of the suburban zone (which determine the city's spatial sizes), cannot be compared unambiguously.

The conclusions in Proposition 2 make intuitive sense. On the one hand, elimination of the suburban-bridge toll tends to make the suburbs more attractive relative to the midcity zone since one of the two previous tolls paid by suburban residents is no longer levied. But the likely increase in the toll on the midcity bridge also increases the attractiveness of the central zone relative to the midcity zone. Given these two attractive forces, the population of the midcity zone should decrease. But the distribution of the relocating population between 
the central and suburban zones is unclear, with a decline in the central zone even a possibility. Therefore, the extent to which cordon tolling shares the first-best regime's ability to shrink $n_{s}$ and thus make the city more compact is unclear. The numerical examples in the next section shed light on this question. Note finally that, with $q_{m}$ larger under cordon tolling, land rent in the midcity must be smaller than under the first-best regime, confirming the tendency seen in $(12)$.

Despite the ambiguity in Proposition 2, the population distributions under the cordon-toll and no-toll regimes can be fully compared. To make this comparison, observe that $t(N-$ $\left.1 / q_{c}-1 / q_{m}\right)-\left[-v\left(q_{m}\right)+v^{\prime}\left(q_{m}\right) q_{m}\right]$ in (19) equals a constant in both the cordon-toll and no-toll cases, with this relationship represented by a downward-sloping curve corresponding to the upper equation-(19) curve in Figure 2. While (20) with $\delta_{m}>0$ again represents the cordon-toll regime, the no-toll case corresponds to (20) with $\delta_{m}<0$. The no-toll regime thus has an equation-(20) curve in Figure 2 that lies above the first-best curve, in the position of the dashed line. As can be seen from the two intersections in the figure, the cordon-toll regime then has a larger $q_{m}$ and a smaller $q_{c}$ than the no-toll regime, yielding a smaller $n_{m}$ and a larger $n_{c}$ under the cordon-toll regime. With $q_{m}$ larger, it then follows that $n_{s}$, the argument of $t$ in the above expressions from (19), must be smaller under the cordon-toll regime. Summarizing yields

Proposition 3. Relative to the no-toll regime, the city's population is more concentrated in the central zone under cordon tolling, with $n_{s}$ and $n_{m}$ smaller and $n_{c}$ larger.

Therefore, like the first-best regime, cordon tolling shrinks the size of the city by reducing $n_{s}$. Moreover population from both non-central zones moves inside the cordon to avoid payment of the toll, with $n_{m}$ smaller along with $n_{s}$. These conclusions stand in contrast to the effects of the first-best regime. The appendix establishes that $n_{s}$ is smaller under the first-best regime than in the absence of tolls, and inspection of Figure 2 shows that $q_{c}$ is lower (and $n_{c}$ is thus higher) in the first-best regime. But the figure indicates that the comparison of $q_{m}$ (and thus $n_{m}$ ) magnitudes is ambiguous. This ambiguity contrasts with the determinate effects seen in Proposition 3. 


\subsection{Numerical example}

To generate a numerical example, the following assumptions are used. The utility from land is given by $v(q)=\alpha q^{\gamma}$, and the bridge-crossing cost is given by $t(n)=\rho+\phi n^{\omega}$. The following parameter values are used: $N=1, I=100, \alpha=4, \gamma=0.5 \rho=0, \phi=0.5$, $\omega=1$, and $\bar{r}=1$. Mathematica could not compute solutions when the exponents $\gamma$ and $\omega$ differed from the assumed values, but variation in the other parameter values was feasible and generated solutions with the same qualitative properties as the solution shown in Table 1.

As seen in Table 1, moving from an untolled city to the first-best regime reduces $n_{s}$, curbing inefficient urban sprawl, while raising both $n_{m}$ and $n_{c}$. While the effect on $n_{m}$ of adopting first-best tolls was ambiguous in general, $n_{m}$ rises along with $n_{c}$ under the assumptions of the example. Land rents move in step with the higher populations in the central and midcity zones.

In moving from the first-best regime to cordon tolling, the toll on the midcity bridge rises substantially, matching the outcome suggested by Proposition 1. In addition, the midcity population falls, as predicted by Proposition 2. Although the destinations of these relocating residents were ambiguous in general, Table 1 shows that population shifts to both the central and suburban zones, with the latter influx increasing the spatial size of the city relative to the first-best outcome. ${ }^{3}$ However, the city remains more compact than in the no-toll regime, as predicted by Proposition 3, a pattern that also appears in the numerical results of Verhoef (2005) and De Lara et al. (2013). Rents in the central and midcity zones rise and fall respectively, in step with the population changes. Note also that, since the rise in $n_{c}$ reduces $n_{m}+n_{s}$,

the first term in the cordon toll formula (15) falls, with the toll increase seen in Table 1 due to the dominant effect of the change in the toll rule (the second term in the formula).

Utility rises by a small amount in moving from the no-toll to the first-best regime, matching other numerical results showing relatively small welfare gains from congestion pricing (see, for example, Verhoef (2005) and Brueckner (2007)). Utility is lower under cordon tolling than under the first-best regime, as expected. However, the cordon toll achieves $92 \%$ of the welfare gain generated by the first-best tolls, a favorable conclusion in line with the findings of previous work in this area (see Mun et al. (2003) and Verhoef (2005)). 
Confirming Proposition 3, Table 1 shows that moving from the no-toll regime to the cordontoll regime raises $n_{c}$ while reducing both $n_{m}$ and $n_{s}$. Therefore, $n_{m}$ changes in opposition directions when moving to the different toll regimes, falling (rising) in moving to the cordontoll (first-best) regime. ${ }^{5}$

\section{Adding Bridge-Capacity Choices}

While bridge capacities were fixed in the preceding analysis, introducing capacity choices generates some additional insights on the effects of cordon tolling. ${ }^{6}$ To make this change, suppose that the conditions leading to the famous self-financing theorem hold (see Small and Verhoef (2007)). In particular, bridge capacity is provided with constant returns to scale, implying that the cost of a bridge with capacity $k$ is $\beta k$, where $\beta>0$. In addition, the bridgecrossing cost is homogeneous of degree zero in traffic volume $n$ and capacity, so that the $t$ function depends on the volume/capacity ratio $n / k$, being written $t(n / k)$. With capacities of $k_{m}$ and $k_{s}$, respectively, the costs of crossing the midcity and suburban bridges are then given by $t\left(\left(n_{m}+n_{s}\right) / k_{m}\right)$ and $t\left(n_{s} / k_{s}\right)$.

To include capacity choices, the costs $\beta k_{s}$ and $\beta k_{m}$ are added to the resource constraint in (3), and the arguments of the $t$ functions are modified as just decribed. In the first-best case, the first-order conditions for $k_{m}$ and $k_{s}$ are given by

$$
\begin{aligned}
& \beta=\left(\frac{n_{m}+n_{s}}{k_{m}}\right)^{2} t^{\prime}\left(\frac{n_{m}+n_{s}}{k_{m}}\right) \\
& \beta=\left(\frac{n_{s}}{k_{s}}\right)^{2} t^{\prime}\left(\frac{n_{s}}{k_{s}}\right)
\end{aligned}
$$

As usual, these conditions yield the self-financing theorem, which can be seen in (21) by multiplying through by $k_{m}$. The resulting LHS expression $\beta k_{m}$ is the cost of capacity, and the RHS expression is equal to $n_{m}+n_{s}$ times the toll per commuter, equal to $\left[\left(n_{m}+n_{s}\right) / k_{m}\right] t^{\prime}$, or total toll revenue from the midcity bridge. Toll revenue thus exactly pays for capacity, an outcome that also holds on the suburban bridge.

Under the cordon-toll regime, the first-order condition for $k_{m}$ is the same as (21). In addition, the toll rule in (16) continues to apply, although the new first-best toll expression 
$\left(\left[n_{m}+n_{s}\right] / k_{m}\right) t^{\prime}\left(\left[n_{m}+n_{s}\right] / k_{m}\right)$ replaces the first term. Since the cordon-toll rule continues to call for larger toll than the first-best rule, it follows from (21) that

$$
\beta k_{m}=\frac{\left(n_{m}+n_{s}\right)^{2}}{k_{m}} t^{\prime}\left(\frac{n_{m}+n_{s}}{k_{m}}\right)<\text { toll revenue from the midcity bridge. }
$$

Therefore, the midcity bridge's toll revenue exceeds its capacity cost, while the suburban bridge generates no toll revenue. Whether the revenue from the cordon toll is sufficient to pay for both bridges is, however, unclear. ${ }^{7}$

In contrast to the choice of $k_{m}$, the first-order condition for $k_{s}$ is altered under the cordontoll regime. The term $(\tau / \lambda)\left(n_{s} / k_{s}^{2}\right) t^{\prime}\left(n_{s} / k_{s}\right)$ is subtracted from the RHS of $(22)$, so that the condition becomes

$$
\beta=\left(\frac{n_{s}}{k_{s}}\right)^{2} t^{\prime}\left(\frac{n_{s}}{k_{s}}\right)\left(1-\frac{\tau}{\lambda n_{s}}\right) .
$$

Since $\tau<0$, the marginal benefit from extra suburban bridge capacity on the LHS of (24) is greater under cordon tolling than under the first-best regime. As a result, the capacity rule points to a larger capacity for the suburban bridge than under the first-best regime. As before, however, this conclusion involves a comparison of rules that contain endogenous quantities that will differ between the regimes. Thus, the intuitive conclusion that the suburban bridge is larger when cordon tolling constrains its toll to be zero is not guaranteed to hold, although the conclusion is highly plausible. Note that, by the same argument, the identical conditions for choice of $k_{m}$ under the two regimes need not imply identical capacities given the presence of other endogenous quantities.

Summarizing yields

Proposition 4. (i) Cordon-toll revenue from the midcity bridge exceeds the cost of optimal capacity for that bridge. (ii) The capacity rule for the suburban bridge under cordon tolling points to a capacity larger than the first-best level, although actual capacity levels, which depend on other endogenous quantities, are not guaranteed to have this relationship. 


\section{Conclusion}

This paper has analyzed cordon tolling using a simple model where space is discrete rather than continuous, with commuting costs incurred only on two congested bridges. While less realistic than the continuous-space frameworks used in previous work, this simple setup allows the derivation of a number of analytical results, which were mostly unavailable to authors relying on more-complex models. The paper derives the rule for the optimal cordon toll, showing that it points to a toll level higher than first-best toll on the midcity bridge. In addition, the analysis shows that cordon tolling leads to a redistribution of population away from the zone immediately outside the cordon, with residents moving to the central zone or to the suburbs. The analysis also shows that cordon tolling raises more revenue than is necessary to pay for the midcity bridge where it is levied, while also tending to increase the capacity of the untolled suburban bridge.

With congestion pricing in the form of cordon tolls now in place in a number of the world's major cities, research on this second-best tolling approach deserves higher priority than before. Use of simple frameworks like the present one may offer new insights about the effects of such toll regimes. 


\section{Appendix}

\section{A1. Proof that $\tau$ is negative}

Equating (7) and the cordon-tolling version of (8) (which has $-\left[\theta+\tau t^{\prime}\left(n_{s}\right)\right] / \lambda$ on the LHS) yields, after canceling $t\left(n_{m}+n_{s}\right)+\left(n_{m}+n_{s}\right) t^{\prime}\left(n_{m}+n_{s}\right)$,

$$
\begin{aligned}
& \underbrace{u-v\left(q\left(r_{m}\right)\right)+q\left(r_{m}\right)\left(r_{m}\right.}_{a}-\tau q\left(r_{m}\right) / \lambda n_{m} q^{\prime}\left(r_{m}\right)) \\
& =\underbrace{u-v\left((q(\bar{r}))+q(\bar{r}) \bar{r}+t\left(n_{s}\right)\right.}_{b}+n_{s} t^{\prime}\left(n_{s}\right)+(\tau / \lambda) t^{\prime}\left(n_{s}\right)
\end{aligned}
$$

The terms $a$ and $b$ in $(a 1)$ are equal since they equal midcity and suburban expenditure, respectively, less the crossing cost and toll incurred on the midcity bridge. Rearranging, $(a 1)$ then becomes

$$
n_{s} t^{\prime}\left(n_{s}\right)+\tau\left(\frac{t^{\prime}\left(n_{s}\right)}{\lambda}-\frac{q\left(r_{m}\right)^{2}}{\lambda n_{m} q^{\prime}\left(r_{m}\right)}\right)=0 .
$$

Since the term multiplying $\tau$ is positive, $\tau$ itself must be negative for $(a 2)$ to hold.

\section{A2. Proof that first-best tolls reduce $n_{s}$ relative to the no-toll regime}

To carry out this demonstration, consider (19) and (20) with $\delta_{s}$ and $\delta_{m}$ set at zero. The resulting equations characterize the first-best regime, while the equations yielded by suppressing the $t^{\prime}$ terms characterize the no-toll regime. Letting the first-best values be denoted by stars and no-toll values by hats, suppose $n_{s}^{*} \geq \widehat{n}_{s}$. Since $t\left(n_{s}^{*}\right)+n_{s}^{*} t^{\prime}\left(n_{s}^{*}\right)>t\left(\widehat{n}_{s}\right)$ then holds, $-v\left(q_{m}\right)+v^{\prime}\left(q_{m}\right) q_{m}$ must be larger in the first-best regime than in the no-toll regime, implying $q_{m}^{*}<\widehat{q}_{m}$ (recall that $q_{s}$ is a constant). The modified versions of (20) then imply

$$
-v\left(q_{c}^{*}\right)+v^{\prime}\left(q_{c}^{*}\right) q_{c}^{*}-\left[t\left(N-1 / q_{c}^{*}\right)+\left(N-1 / q_{c}^{*}\right) t^{\prime}\left(N-1 / q_{c}^{*}\right)\right]>-v\left(\widehat{q}_{c}\right)+v^{\prime}\left(\widehat{q}_{c}\right) \widehat{q}_{c}-t\left(N-1 / \widehat{q}_{c}\right) . \quad(a 3)
$$

The RHS of (a3) is decreasing in $q_{c}^{*}$ and the LHS is decreasing in $\widehat{q}_{c}$, while the RHS is smaller than the LHS at a common value of $q_{c}^{*}$ and $\widehat{q}_{c}$. Therefore, satisfaction of the inequality 
requires $q_{c}^{*}<\widehat{q}_{c}$. Given $n_{s}=N-1 / q_{c}-1 / q_{m}$, this inequality along with $q_{m}^{*}<\widehat{q}_{m}$ from above then imply $n_{s}^{*}<\widehat{n}_{s}$, contradicting the assumption that $n_{s}^{*} \geq \widehat{n}_{s}$ holds and thus establishing the contrary. 
Table 1

Numerical Example

\begin{tabular}{lrrr}
\hline \hline & Untolled & First-best regime & Cordon tolling \\
\hline suburban toll & 0 & 0.1482 & 0 \\
midcity toll & 0 & 0.2940 & 0.4403 \\
central rent $\left(r_{c}\right)$ & 1.1526 & 1.2838 & 1.2865 \\
midcity rent $\left(r_{m}\right)$ & 1.0514 & 1.0800 & 1.0410 \\
central pop. $\left(n_{c}\right)$ & 0.3321 & 0.4120 & 0.4138 \\
midcity pop. $\left(n_{m}\right)$ & 0.2764 & 0.2916 & 0.2709 \\
suburban pop. $\left(n_{s}\right)$ & 0.3915 & 0.2963 & 0.3152 \\
utility $(u)$ & 103.6744 & 103.6963 & 103.6946 \\
multiplier $(\tau)$ & $\mathrm{NA}$ & $\mathrm{NA}$ & -0.0208 \\
\hline \hline
\end{tabular}




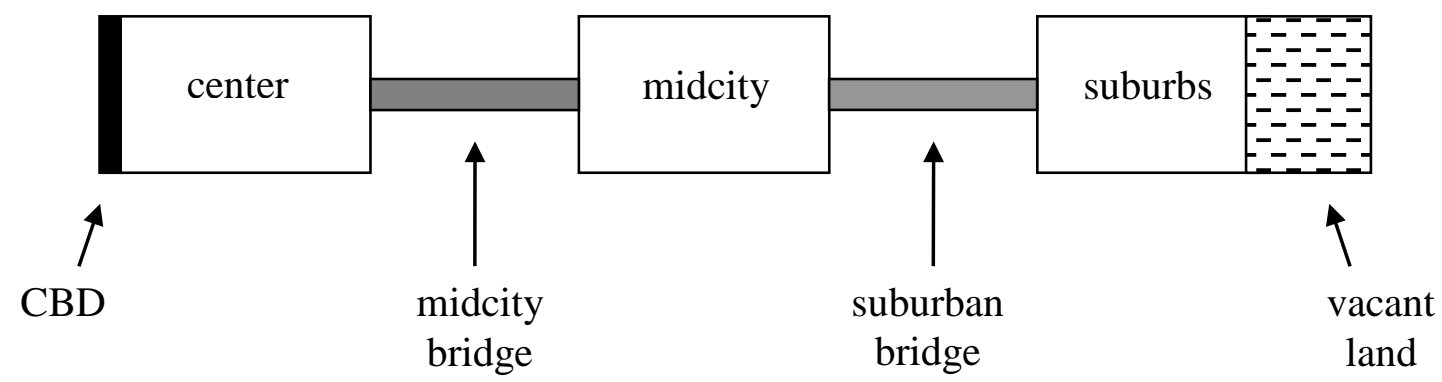

Figure 1: City map 


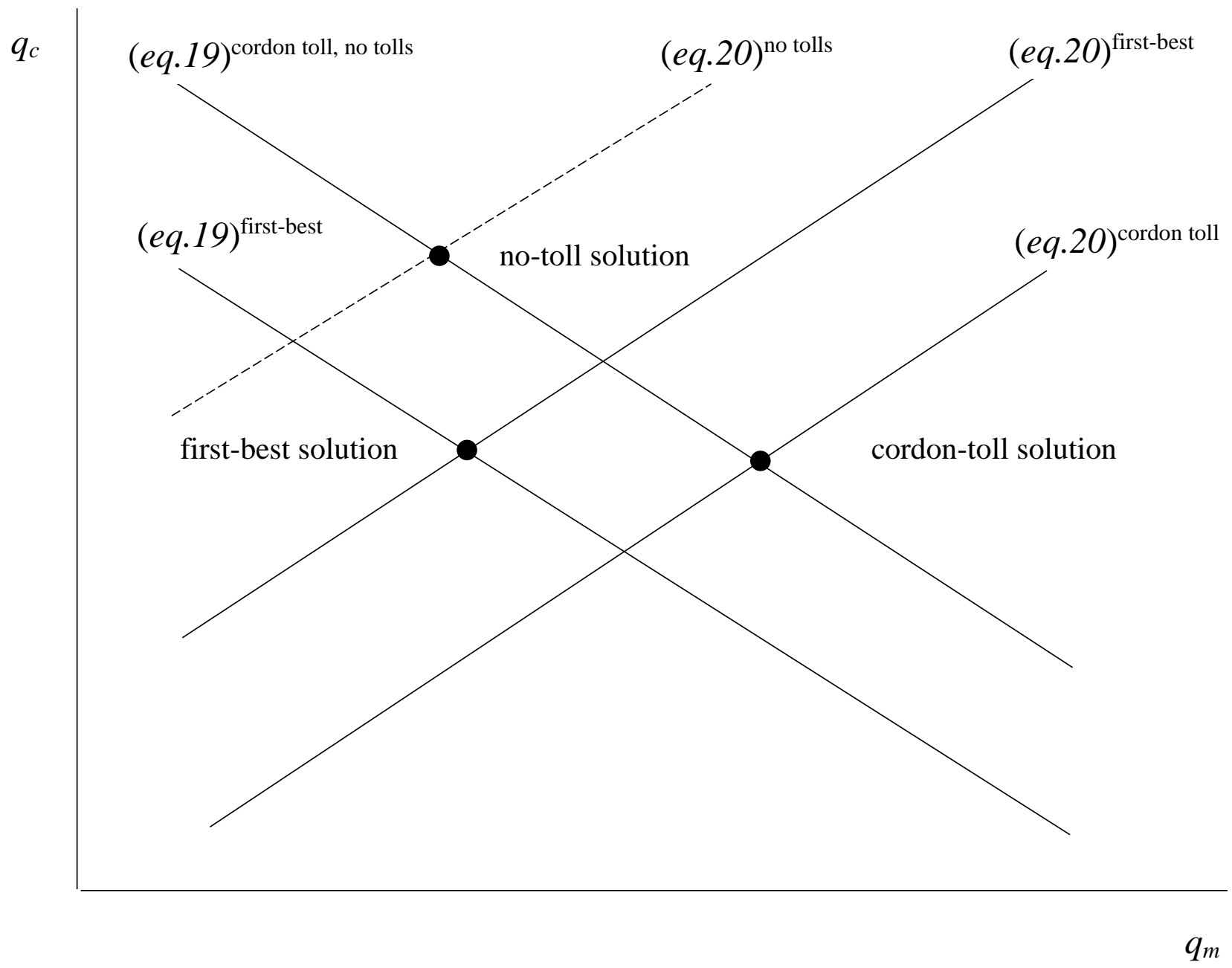

Figure 2: Land consumption levels in the three regimes 


\section{References}

Arnott, R.J., 1979. Unpriced transport congestion. Journal of Economic Theory 21, 294316.

Brueckner, J.K., 2007. Urban growth boundaries: A effective second-best remedy for unpriced traffic congestion? Journal of Housing Economics 16, 263-273.

Brueckner, J.K., Helsley, R.W., 2011. Sprawl and blight. Journal of Urban Economics 69, 205-213.

Brueckner, J.K., 2014. Decentralized road investment and pricing in a congested, multijurisdictional city: Efficiency with spillovers. Unpublished paper, UC Irvine.

De Lara, M., de Palma, A., Kilani, M., Piperno, S., 2013. Congestion pricing and long term urban form: Application to Paris region. Regional Science and Urban Economics 43, 282-295.

Ho, H.W., Wong, S.C., YAng, H., Loo, B.P.Y., 2005. Cordon-based congestion pricing in a continuum traffic equilibrium system. Transportation Research Part A 39, 813834.

Kanemoto, Y., 1976. Cost-benefit analysis and the second-best land use for transportation. Journal of Urban Economics 4, 483-503.

Mills, E.S., De Ferranti, D.M., 1971. Market choices and optimum city size. American Economic Review, Papers and Proceedings 61, 340-345.

Mun, S., Konishi, K., Yoshikawa, K., 2003. Optimal cordon pricing. Journal of Urban Economics 54, 21-38.

Pines, D., SAdKA, E., 1985. Zoning, first-best, second-best and third-best criteria for allocating land for roads. Journal of Urban Economics 17, 167-183.

Pines, D., SAdKA, E., 1986. Comparative statics analysis of a fully closed city. Journal of Urban Economics 20, 1-20.

Small, K.A., Verhoef, E.T., 2007. The Economics of Urban Transporation. Routledge, Abington, UK.

Solow, R.M., 1971. Land use in a long narrow city. Journal of Economic Theory 3, 430-447.

Verhoef, E.T., 2005. Second-best congestion pricing schemes in the monocentric city. Jour- 
nal of Urban Economics 58, 367-388.

Wheaton, W.C., 1998. Land use and density in cities with congestion. Journal of Urban Economics 43, 258-272. 


\section{Footnotes}

*I am indebted to Se-il Mun for some very helpful suggestions. Any errors or shortcomings, however, are mine.

${ }^{1}$ For a treatment of cordon tolls from a transportation-engineering perspective, see Ho, Wong, Yang and Loo (2005).

${ }^{2}$ Alternatively, the midcity toll could be set at zero, with a positive toll on the suburban bridge. However, the reverse pattern is a more realistic representation of actual cordon tolls, which are levied close to the CBD.

${ }^{3}$ Since other parameter values lead to solutions where the $n_{c}$ values under the two regimes differ only in the fourth or fifth decimal place, it appears that outcomes where $n_{c}$ is smaller under cordon tolling could emerge, possibly under different functional-form assumptions.

${ }^{4}$ The results of De Lara et al. (2013) show much larger differences in the utility gains under the two regimes.

${ }^{5}$ Note that since Propositions 2 and 3 imply that $n_{m}$ falls in moving from the no-toll regime to the cordon-toll regime but then rises in moving further to the first-best regime, no comparison of $n_{m}$ 's magnitudes in the no-toll and first-best regimes follows, confirming the ambiguous conclusion mentioned earlier.

${ }^{6}$ In a similar fashion, road width at each distance from the CBD is chosen optimally in the model of De Lara et al. (2013). This feature of their analysis may account for the relatively large disparity in utility gains between the first-best and cordon-toll regimes.

${ }^{7}$ Mathematica was unable to converge to a solution for the cordon-toll problem with capacity choices. 